\title{
RELIABILITY ENGINEERING ON FEEDING SYSTEMS IN AUTOMATIC MACHINES OF HIGH PRODUCTION CAPACITY
}

\author{
Ryspek Usubamatov', Thoguluva Raghavan Vijayaram² ${ }^{2}$ Usubamatova A. ${ }^{3}$ \\ ${ }^{1,3}$ School of Manufacturing Engineering, University Malaysia Perlis, Malaysia \\ ${ }^{2}$ Faculty of Manufacturing Engineering, University Teknikal Malaysia, Melaka, Malaysia \\ Email: 'ryspek@unimap.edu.my
}

\begin{abstract}
Predicting reliability has become an important engineering science and involves complex mathematical relationships and calculations. The reliability of an automated high-speed production line with all its complex mechanical and electronic components is also important, as its failure can result in major economic losses to the manufacturer. This paper reviews about the emphasis of reliability engineering on the feeding system and element in automatic machines. It also discusses about the calculation of the reliability parameters on the feeding unit by applying the probability theory. Various formulae and derived to calculate the limit size or the chute width and probability or the part sticking into the shut. Besides, a case study has undertaken to calculate the value of probability.
\end{abstract}

Keywords: Reliability, feeding system, automatic machines, rhythmic feeders, chutes, workflow, probability and statistical theory.

\section{INTRODUCTION}

Reliability is defined as the probability that a product will perform its intended function in a given environment and for a specified period, without failure. Reliability is classified into two types. Among them, series reliability is an example of the feeding system in automatic machines of high production capacity. Another type is called as parallel reliability. Automatic machines of high production capacities have complex feed units and transport system at the outlet of the production systems to transfer the processed parts to the storage units. These fulfill the functions of feeding, output, accumulation, linking and separation of the workflow [1]. The existing developed transport and storage system for feeding in the automatic machines contain the set of devices like hoists, conveyors, distributors which channels and guide ways. The principle of functioning of these devices are based on the forced displacement of parts, by the action of forces of gravity and by the mixed principle of the parts displacement concept, which combines the forces of gravity and externally applied forces for the transportation of parts in the transport and storage systems [1,2] . The abovementioned principle can be observed in majority of the cases in the system. The motion of the parts is accomplished in different various by pieces, intermittently or by continuous flow depending on the requirement of productive units. The diverse variants of the motion of the parts are achieves with the aid of the devices for group displacement in the cells of the transporting organ by the application of different type devices lie stoppers, rhythmic feeders and so on. In practice, the majority of the cases for the displacement of parts utilize their properties for the sliding and rolling, which facilitates in many aspects for transportation and feeding simplifies and reduces the cost of them [3]. The displacement of parts by sliding or rolling, consequently in the tentative position is produced in the guides, chutes and channels of those having the strictly defined form of its cross section as a rule of rectangular. All the above are seated from the standardized elements like guide bars, supporting plates and tapes secured by the screws $[4,5]$. Guide bars, shuts, feeding channels and so are made from different materials like plastics, woods and steels depending on the requirement by the industries. These can be bent, hence makes possible to rapidly build them into the systems for easy installation and dismantling and to connect the feeding systems with the automatic machines and the production lines [6]. Most automatic machines have simple construction and however, experience of operating the work in these machines and in the line for processing parts testifies on the low reliability of the simple mechanism of transport, feeding and storage system. This bears out the fact that question of the reliability on these simple system designed by the engineers and scientist don't give a sufficient attention, considering this question superfluous since the transport, feeding and storage system do not relate to the basic technological equipment that generating parts.

\section{SUBSTANTIATIONS OF THE SELECTED DIRECTION OF THE RESEARCH WORK ON RELIABILITY PROBLEMS}

In the automatic machines of high productivity having the duration of working cycle at range of several seconds, questions on the reliability of transport, feeding and storage systems acquire special paramount importance [7] . For example, the simplest chute systems in spite of their simplicity in design remain some of the most unreliable elements of the transport system in the automatic machines and in the production lines [8]. 
Studies on the reason for stops, brakes and their comprehensive analysis have made it possible to determine the nature of their appearance and hence to develop design and improve the technological recommendations in order to increase the fitness of work of the elements of systems. Sticking of parts into the shuts and feeders may be due to the following group of factors like cyclically operative factors, monotonically acting factors and also on the design factors like the imperfection of the elements by construction and devices operating mechanisms. The reason for the stop, fails and brakes of the system obey the laws of the probability theory [9]. As it is evident that the proposed normative given and the formulas for the calculation of chute dimensions does not considered the factors of the sizes speed of the parts design parameter of the chutes, and also the dynamics of the motion of the parts, where can occur their collision, which in turn draws either sticking or releasing the parts [9, 10]. Existing normative data are presented and the analysis shows that it has not taken into account of the actual spread of the sizes of the moving parts and the parameters of chutes and conveying appliances, which naturally affects on the reliability of the parts displacement. Some authors propose the limit of the sizes that guarantee the reliable transportation of parts into shut. They propose the below mentioned formula (1) to determine the limit of size of the chute width with reference to Fig.1.(a), represented as:

$W=\sqrt{\frac{H^{2}+D^{2}}{1+f^{2}}}$

Where $\mathrm{H}=$ height of the part, $\mathrm{D}=$ =width of the part, $\mathrm{f}=$ coefficient of friction and $W=$ chute width
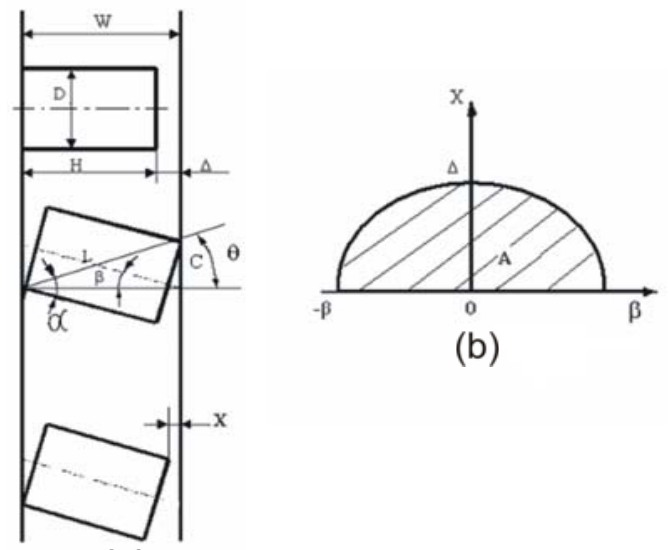

(b)

(a)

Fig. 1. Moving part displacement diagram

Studies on the design parameters of the elements for transport systems and parts show that their sizes are the random variables, distributed in a specific range [11].
Processing the results of measurement by statistical and probabilistic methods shows that the sizes are distributed according to the normal law. The density of probability distribution of normal law is expressed in the form as $f(x)$, and defined by the formula (2).

$$
f(x)=\frac{1}{\sqrt{2 \Pi_{x}}} e^{\frac{\left(x_{i}+m_{x}\right)^{2}}{{ }^{2} \sigma_{x}^{2}}}
$$

Where $f(x)$ is the density of the probability distribution of the specific values if random variable, $x$ or theoretical frequency, $x_{0}=$ specific possible values of random variable, $m_{x}=$ mathematical expectation of the random variable, $\sigma_{x}=$ deviation of the random variable and $\mathrm{e}$ (base of the natural logarithm) $=2.718$

\section{CALCULATION OF RELIABILITY PARAMETERS ON THE MOVING PARTS IN FEEDING SYSTEMS}

During the motion of the parts into the feeding systems, their stops, accumulates and collides which leads to the failure of the systems due to sticking of parts into the shuts, channel and guides. Failure may be influenced by the factors like shape, geometry of parts, accuracy of parts and feeding element, speed of the moving parts and chaotic character of the part motion. Let us consider the reliability motion of the sliding of a simple rectangular part into an inclined shuts, shown in Figure-1. The motion of the parts into the shut is presented as contacts of slide part with the shut board and part can swing and can stick into shut [12]. This citation depends from the geometrical sizes of the parts, sizes of the shut width and from the path swing [13]. The part motion into a shut is chaotic, their swing in random, also the sizes of the parts and the size of the shuts width are also random and therefore it is possible to calculate the probability dependencies of the parts sticking into a shut by laws of probability. The part sticking into the shut depended from the part swing and its contact with the shutboards;

- appearing of the definite size of part height, $\mathrm{H}$

- appearing of the definite size of the part width, $D$

- appearing of the definite size of the shut width, $\mathrm{W}$

because all the events are random: part sizes, shut width, part swing and are accidentally independent value, therefore the probability of the part sticking can be calculated as the multiplication of the probabilities event according to the theorem of the probabilities theory about the appearance of random

event by the formula (3)

$\mathrm{P}=\mathrm{P}_{\mathrm{SW}}{ }^{*} \mathrm{P}_{\mathrm{H}}{ }^{*} \mathrm{P}_{\mathrm{D}}{ }^{*} \mathrm{P}_{\mathrm{W}}$ 
Where

$P=$ probability of the part sticking into shut

$\mathrm{P}_{\mathrm{SW}}=$ probability of the part swinging and contacts with the shutboard

$P_{H}=$ probability appearing of the definite size of the part height, $\mathrm{H}$

$P_{D}=$ probability appearing of the definite size of the part width, $D$

$P_{w}=$ probability appearing of the definite size of the shut width, $W$

In such a way to calculate the probability of a part sticking into a shut, it is necessary to know the probabilistic values of the factors in the formula (3).

\section{DEFINITION OF THE PROBABILISTIC VALUE OF THE PART SWINGING AND ITS CONTACTS WITH SHUT BOARDS}

Normally, the motion of the part into a shut as chaotic and at any position it has equal probability that is shown in Fig.1.(a) The part orientation and its contacts with the shut boards is expressed by the possible probabilities values as $X$ and $\beta$. $X$ is distance from the parts side to the shut board and $\beta$ is an angle created by the part axis that is perpendicular to the shut board. $\beta$ is also defined as a part swing angle [14]. The value of $X$ and $\beta$ have continuous uniform distribution. $X$ is distributed on the interval from 0 to $\mathrm{W}-\mathrm{H}$ but in 2 directions to the left and right boards [14, 15]. This leads to the part swinging which is defined by an angle $\beta$ and its value changes on the interval from $\theta$ toa, but two directions as $-\beta$ to $+\beta$ by clockwise and anticlockwise directions correspondingly shown in Figure1. The angle $\alpha$ is the angle between the parts diagonal and its axis and the angle, $\theta$ is the angle between the parts diagonal that is perpendicular to the shut board when the parts contact with the shut board. So the density of the uniform distribution random variable, $\mathrm{X}$ is expressed by the formula (4)

$f(x)=\left(\frac{1}{2(W-H)}\right)$

Where $\mathrm{W}$ is shut width, $\mathrm{H}$ is the part height and number 2 in the above formula has 2 directional distributions as $X €$ $(0, W-H)$ and $\beta \in|\theta, \alpha|$. Hence $f(x)=0$ by $X \in(0, W-H)$ or $f(\beta) \Theta|\theta, \alpha|$.

With the reference to Fig.1.(b), let us consider a place $X_{0}$ having the area of possible meaning random values, $X$ and $\beta$ orientation and position of a part into a shut is possible if the next condition is feasible as $X=W-\cos \beta$ where $L$ is a part diagonal (i.e) the random point with coordinate $X$ and $\beta$ falls in the area $A$ which is shown by the hatched lines in Figure-2. Hence, the probability distribution of any parts position into the shut is expressed by $g(\beta)=f(x)|J|$ where $J=f(x)$ is called as the jacobian transformation function. Hence, $J=(W-L \cos \beta)=L \sin \beta$ then $g(\beta) \frac{1}{2(W-H)}(L \sin \beta)$

The probability of any parts position into the shut is expressed by

$$
\begin{aligned}
P_{S W} & =\int_{-\beta}^{+\beta} g(\beta) d \beta=2 \int_{0}^{\beta} \frac{L \sin \beta}{2(W-H)} \cdot D \beta \\
P_{S W} & =\left[\frac{2 L \cos \beta}{2(W-H)}\right]_{0}^{\beta} \text { Or } P_{s w}=\frac{-L(\cos \beta-\cos 0)}{(W-H)} \\
\therefore P_{S W} & =\left[\frac{-L(\cos \beta-1}{(W-H)}\right], \text { But } \beta=\alpha-\theta, L=\sqrt{H^{2}+D^{2}}
\end{aligned}
$$

We know that, $\cos (\alpha-\theta) \cos \alpha \cos \theta+\sin \alpha \sin \theta$

$$
\text { So } \cos \alpha=\left[\frac{H}{L}\right]=\left[\frac{H}{\sqrt{H^{2}+D^{2}}}\right], \cos \theta=\left[\frac{W}{L}\right]=\frac{W}{\sqrt{H^{2}+D^{2}}}
$$

similarly,

$$
\begin{gathered}
\sin \alpha=\left[\frac{D}{L}\right]=\left[\frac{H}{\sqrt{H^{2}+D^{2}}}\right], \sin \theta=\left[\frac{W}{L}\right]=\frac{W}{\sqrt{H^{2}+D^{2}}} \\
\therefore \sin \theta\left[\frac{\sqrt{H^{2}+D^{2}-W^{2}}}{\sqrt{H^{2}+D^{2}}}\right]=\sqrt{\frac{H^{2}+D^{2}-W^{2}}{H^{2}+D^{2}}}=\sqrt{\frac{H^{2}+D^{2}}{H^{2}+D^{2}}-\frac{W^{2}}{H^{2}+D^{2}}}
\end{gathered}
$$

$\sin \theta=\sqrt{1-\left(\frac{W^{2}}{H^{2}+D^{2}}\right)} \quad$ After substitution and transformation the formula of the part swinging into the shut will have the expression as follows:

$P_{S W}=\frac{-\left[H W+D \sqrt{H^{2}+D^{2}-W^{2}}-H^{2}-D^{2}\right]}{\left(\sqrt{H^{2}+D^{2}}\right)(W-H)}$

The probabilistic function of distribution of sizes $H, D$ and $W$ is defined by integral dependencies on the base of the formula and their density of probability distribution have expression as $F(B)$ and $P(B+\delta)$

Hence $F(B)=\frac{1}{\sigma_{B} \sqrt{2 \pi}} \int_{-\infty}^{\infty} e^{-\frac{\left(B-m_{B}\right)}{2 \sigma^{2} B}} d B$

Where the parameter must be replaced by $\mathrm{H}, \mathrm{W}$ and $\mathrm{D}$. If it takes some interval, it is possible to calculate the probability of appearing of the sizes $\mathrm{H}, \mathrm{D}$ and $\mathrm{W}$ by the next consecutive. 


\section{CASE STUDY}

For the part and shut, there are next data: shut width $\mathrm{W}=330 \pm .3 \mathrm{~mm}$, part height $\mathrm{H}=30 \pm 0.1 \mathrm{~mm}$, part width $D=150.1 \mathrm{~mm}$, and all the dimensions have normal distribution. The probability of appearing the critical size of the shut width when the part can stick is 0.1 , The probability of appearing the sizes that less than of the part length and width is 0.05 and the coefficient of friction is changing on an interval from $0.05-0.15$. In the feeding system can be located $\mathrm{N}=20$ parts, that move separately. An industrial machine has cycle time $\mathrm{T}=3$ seconds. Find the average time when feeding system of the machine will work without fails.

Solution: The critical size of the shut width is defined by formula (1) with minimum size of part height and width and meaning of coefficient of friction $f=0.1$ on an average.

$$
\mathbf{W}=\sqrt{\frac{\mathbf{H}^{2}+\mathbf{D}^{2}}{1+\mathbf{f}^{\mathbf{2}}}}=\sqrt{\frac{29.9^{2}+\mathbf{1 4 . 9}^{\mathbf{2}}}{1+\mathbf{0 . 1}^{2}}}=33.24
$$

The maximum size of the part height when a part is sticking can be calculated with the maximum shut's width and minimum part's width and meaning of coefficient of friction $f=0.1$

$\mathbf{H}=\sqrt{\mathbf{W}^{\mathbf{2}}\left(\mathbf{1}+\mathbf{f}^{\mathbf{2}}\right)-\mathbf{D}^{\mathbf{2}}}=\sqrt{\mathbf{3 3 . 3}^{\mathbf{2}}\left(\mathbf{1}+\mathbf{0 . 1}^{\mathbf{2}}\right)-\mathbf{1 4 . 9}^{\mathbf{2}}}=29.96$

The maximum size of the part's width when a part is sticking can be calculated with the maximum shut width and minimum part's height and meaning of coefficient of friction $f=0.1$

$\mathrm{D}=\sqrt{\mathrm{W}^{\mathbf{2}}\left(\mathbf{1}+\mathbf{f}^{\mathbf{2}}\right)-\mathrm{H}^{\mathbf{2}}}=\sqrt{\mathbf{3 3 . 3 ^ { 2 }}\left(\mathbf{1}+\mathbf{0 . 1}^{\mathbf{2}}\right)-\mathbf{2 9 . 9}^{\mathbf{2}}}=15.03$

Critical sizes of part's height is $H=29.9-29.96$, critical sizes of part's width is $D=14.9-15.03$, critical sizes of shut's width is $W=33.24-33.3$. The probability of the critical sizes of part's height and width and Shute width of appearing calculated next. We have $\mathrm{m}_{H}=30, \sigma_{H}=0.2 / 6=$ $0.0333, \mathrm{mB}=15, \sigma B=0.2 / 6=0.0333, \mathrm{~m}_{\mathrm{w}}=33, \sigma_{\mathrm{w}}=0.6 / 6=$ 0.1

$$
\begin{aligned}
& P(29.96-29.9)=\frac{1}{0.0333 \sqrt{2 \pi}} \int_{29.9}^{29.96} \mathrm{e}^{-\frac{(29.96-30)^{2}}{2^{\star} 0.0333^{2}}} \mathrm{dH}=\mathbf{0 . 1 2 3 8} \\
& P(15.03-14.9)=\frac{1}{0.0333 \sqrt{2 \pi}} \int_{14.9}^{15.03} \mathrm{e}^{-\frac{(15.03-15)^{2}}{2^{\star} 0.0333^{2}}} \mathrm{dD}=\mathbf{0 . 8 1 4 6} \\
& P(33.3-33.24)=1-\frac{1}{\mathbf{0 . 0 3 3 3} \sqrt{2 \pi}} \int_{33.24}^{33.3} \mathrm{e}^{-\frac{(33.3-33.0)^{2}}{2^{\star} 0.0333^{2}}} \mathrm{dW}=\mathbf{0 . 2 7 5 6}
\end{aligned}
$$

The probability of the part swing, Psw is defined by formula (5) and after substitution of all the initial data yields $e=33+30=63, \quad-k^{2}=-(15 / 63)^{2}+3 / 63, k=0.0952$, $c=0.1428$,

$P_{S w}=\frac{1}{2 * 63 * 0.0952} \ln \left|\frac{0.1428[(15 / 63)-0.0952]-\left[(15 / 63)^{2}-0.0952^{2}\right]}{0.1428[(15 / 63)+0.0952]-\left[(15 / 63)^{2}-0.0952^{2}\right]}\right|$ $\mathrm{P}_{\mathrm{SW}}=0.5398$

From the previous, the probability of the part stick is defined by formula (3),

$\mathrm{P}=\mathrm{P}_{\mathrm{SW}}{ }^{*} \mathrm{P}_{\mathrm{H}}{ }^{*} \mathrm{P}_{\mathrm{D}}{ }^{*} \mathrm{P}_{\mathrm{W}}$

After substituting the values of $\mathrm{P}_{\mathrm{SW}}, \mathrm{P}_{\mathrm{H}}, \mathrm{P}_{\mathrm{D}}$ and $\mathrm{P}_{\mathrm{w}}$, then $\mathrm{P}=$ $0.5398^{*} 0.1238^{*} 0.8146^{*} 0.2756=0.015$

The probability to calculate the 20 parts that stick into feeding system is defined by a general formula (6) as follows:

$\mathrm{Pn}=1-(\mathrm{P})^{\mathrm{n}}$

Here in our case, $n=20$ and hence substituting it, we get

Therefore, $P_{20}=1-(1-0.015)^{20} \approx 0.0002$

\section{RESULTS AND DISCUSSION}

The probability of parts stick into the feeding system of the machine will have 2 cases per 1000 cycles or 2 fails per 3000 seconds. In average, the feeding system of a machine will fail after 25 minutes or the feeding system of machine will work without fail 25 minutes. Research on the reliability of the feeding systems of machines depends on the combined geometry of the machine unit and indexes of its reliability. Such approach provides the chances and possibilities to the design engineers to calculate the reliability of the machines via geometrical dimensions of their units. The formulae for calculating the probability of parts sticking into a feeding system of a complex industrial automatic machine allows us to develop methodologies for calculating the reliability of feeding system as a function of its geometrical dimension and sizes.

\section{CONCLUSION}

- The real part and real shut should be defined by the deviation of its sizes to obtain the dependencies of their distributions.

- Define the limit sizes of the part and the shut width when the part is sticking.

- Calculate the probability of a part swinging and contacting into shut boards.

- Calculate the probability of apart and shut limit sizes that appears which can create the conditions for 
parts sticking into the shuts.

- The calculation of the reliability of the feeding systems should respond to the productivity increase of the machines.

- The used formulae are concerned to the simple rectangular shape of the parts and the methodology allows us to decide more complex problems.

\section{REFERENCES}

[1] Ronald E. Walpole, Raymond. H. Myres, Sharon L. Myres, 1998, Probability and Statistics for Engineers and Scientist (Prentice Hall international Inc) , 78-87

[2] Kalpakjan, S,1984, Manufacturing Process for Engineering Materials, Reeding Massachusetts, (Addison-Wesley, USA), 90-95

[3] Kales. P.,1997, Reliability: For Technology Engineering and Management, (Prentice Hall), 102-108

[4] reason, W.A, C.F. Coombs, Jr and R.Y. Mors, 1996, Handbook of Reliability Engineering and Management, 2nd Edition, (Mc Graw Hill), 54-67

[5] Lewis E.E,1995, Introduction to Reliability Engineering 2nd Edition, (John Wiley), 108-111.

[6] Grant E.L. and R.S. Leavenworth,1997, Statistical Quality Control, (Mc Graw Hill), 143-148

[7] Essayed, E.A.,1996, Reliability Engineers, (Addison-Wesley), 45-54

[8] Clement R.B,1991, The Handbook of Statistical Method in Manufacturing, (Prentice Hall), 102-117.

[9] Bently, J.R.,1999, Introduction to Reliability and Quality Control, 2nd Edition, (Addison-Wesley), $125-128$

[10] Miller I., Freud J.e.,1985, Probability and Statistics for Engineers 3rd Edition, (Eaglewood Cliffs,
Prentice Hall), 78-89.

[11] Amstead, B.H., Oswald, Philip F. and Begemen M.L.,1979, Manufacturing Process, 7the Edition, (John Wiley \& Sons New York), 98-109.

[12] Degarmo, E. Paul, Black, J.Temple and Kohser, R.,1984, Materials and process in Manufacturing, 6th Edition, (Macmillan), 67-75

[13] Groove, M.P,1980, Automation Production Systems and Computer-Aided Manufacturing, (Eaglewood Cliffs, NJ, Prentice Hall), 56-59

[14] Ronald G.A, and CharlesR.S.,1993, Modeling and Analysis of Manufacturing Systems, (John Wiley \& Sons, New York), 89-97

[15] Buzacott J.,1972, Method of Reliability Analysis of Production System Subject to Breakdown, Operation and Reliability, Daniel Gronchko Edition (Gordon and Breach Science NY), 211-213.

Ryspek Usubamatov is a Doctorate in Automation of

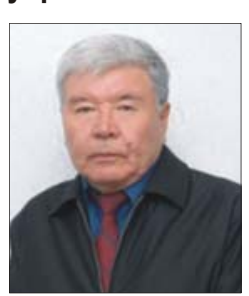
Manufacturing Processes from Moscow State Technical University and a Professor at the School of Manufacturing Engineering, University Malaysia Perlis. He has received more than 60 patents of inventions in area of automation of manufacturing processes, machine tools design, robotics, industrial automated transport systems and technological processes. His fields of interests include Theory of Industrial and Manufacturing Productivity, Metal Cutting Manufacturing Processes, Industrial Machines Reliability, Automation of Manufacturing Processes and Inventions in different areas of human activity, CNC/CAD/CAM and Multi-axis Gear Machining on CNC Machining Centres. 